BULLETIN Bulletin hispanique

HispanIQUE Université Michel de Montaigne Bordeaux

112-1| 2010

Actes du Colloque « langue, littérature, littéralité »

\title{
Une poétique de l'interlocution dans les autos sacramentales de Tirso de Molina
}

Isabelle Bouchiba-Fochesato

\section{(2) OpenEdition}

Journals

Édition électronique

URL : http://journals.openedition.org/bulletinhispanique/1112

DOI : 10.4000/bulletinhispanique. 1112

ISSN : 1775-3821

Éditeur

Presses universitaires de Bordeaux

Édition imprimée

Date de publication : 1 juin 2010

Pagination : 113-129

ISBN : 978-2-86781-692-5

ISSN : 0007-4640

Référence électronique

Isabelle Bouchiba-Fochesato, « Une poétique de l'interlocution dans les autos sacramentales de Tirso de Molina », Bulletin hispanique [En ligne], 112-1 | 2010, mis en ligne le 01 juin 2013, consulté le 19 avril 2019. URL: http://journals.openedition.org/bulletinhispanique/1112 ; DOI : 10.4000/

bulletinhispanique. 1112 


\title{
Une poétique de l'interlocution dans les autos sacramentales de Tirso de Molina
}

\author{
Isabelle Bouchiba-Fochesato \\ Université Michel de Montaigne Bordeaux
}

Cet article tente de démontrer l'existence d'une véritable poétique de l'interlocution dans les autos sacramentales de Tirso de Molina, directement liée à celle mise en jeu dans les comedias du même auteur, c'est-à-dire l'existence d'une finalité, propre aux autos, de l'alternance des pronoms d'adresse.

Este artículo intenta demostrar que la alternancia de los tratamientos en los autos sacramentales de Tirso de Molina desempeña un papel clave en la economía global de las obras en total coherencia con el sistema que existe en las comedias del mismo autor.

The author of the article aims at proving the existence of a true poetics of interlocution in Tirso de Molina's 'Autos Sacramentales', in direct link with the one in progress in the 'Comedias' by the same author. In other words, that there is a specific goal in the use of the addressing pronouns in the 'Autos'.

Mots-clés : Autos sacramentales - Interlocution - Siècle d'Or - Tirso de Molina Thêâtre.

E TeRme hommage tel que le définit le Petit Robert et tel qu'employé ici,
a pour sens « témoignage de respect, d'admiration, de reconnaissance ». Qu'il me soit permis ici de dire - trop brièvement - dans quelle mesure

$B H i$, Tome 112, nº 1 - juin 2010 - p. 113 à 129. 
respect, admiration, reconnaissance et j'ose l'ajouter, amitié, constituent, de manière absolument indissociable, la formule exacte du sentiment qui est le mien à l'heure d'écrire ces lignes pour Nadine Ly. Nadine Ly n'a pas été mon seul maître, c'est-à-dire celui ou celle qui forme et transforme, qui transmet une méthode, qui oriente une carrière. Elle n'a pas été la seule, mais elle est certainement celle à qui, au final, je dois le plus et celle qui continue, encore à ce jour, à me former et à me transformer. Elle est, en outre, pour moi l'image d'une Université que j'apprécie et que je comprends. L'admiration que j'éprouve pour Nadine Ly est intellectuelle et humaine, le respect que j'ai pour elle tient bien sûr à son savoir, à son savoir-transmettre, mais plus généralement encore à son savoir-être. Ma reconnaissance est sans borne.

Mon travail de thèse, intitulé "La poétique de l'interlocution dans les comedias de Tirso de Molina "s'est employé à démontrer d'une part que l'alternance des pronoms d'adresse dans le théâtre Tirsien élaborait des protocoles interlocutifs spécifiques participant de la structuration des personnages eux-mêmes - ils sont un élément déterminant de leur idiolectemais aussi des situations dramatiques - scènes de séduction, scènes familiales, scènes de jalousie etc. - et d'autre part qu' elle participait de la hiérarchisation interne à la comedia, orientée vers une relation inférieur/supérieur. Il se fondait aussi sur le principe que les contraintes qui s'appliquent en termes de choix des allocutifs dans les dialogues de théâtre s'affranchissent des règles de courtoisie en vigueur en cette seconde moitié du XVII ${ }^{e}$ pour mieux jouer sur le double tableau de ces mêmes règles de la sociolinguistique et de celles de l'héritage poétique des siècles passés (théâtre pré-lopesque, mais aussi poésie courtoise médiévale voire tradition issue du théâtre latin comme dans le cas du tutoiement entre maître et valet) pour élaborer un système interne à la comedia.

Ce travail trouvait sa source et son inspiration dans la thèse de Nadine Ly, La poétique de l'interlocution dans le théâtre de Lope de Vega et il m'a donc paru tout naturel d'en proposer une extension dans un article visant avant tout à rendre hommage à celle qui a dirigé ma thèse et qui m’a également inculqué une méthode de travail. Cette extension s'imposait d'elle-même : je n'avais pas traité dans ma thèse le cas des autos sacramentales. Cela étant, cet article ne traitera pas de tous les échanges dialoguaux des autos. C'est en effet un véritable mémoire qu'il faudrait y consacrer. Je me limiterai donc

1. Nadine Ly, La poétique de l'interlocution dans le théâtre de Lope de Vega, Institut d'Études Ibériques et ibéro-américaines de l'Université de Bordeaux, 1981. 
ici aux dialogues mettant en présence les personnages surnaturels (Dieu, le Diable, des anges) ou leurs adjuvants déclarés et les sujets sur lesquels ils tentent d'agir (humains ou non anthropomorphes), afin de mettre en évidence l'originalité des autos sacramentales.

De Tirso de Molina, ne nous sont parvenus que 6 autos sacramentales. Que le dramaturge n'en ait pas composé d'autres ou que ces pièces se soient perdues au fil des siècles importe peu ici. Il semble cependant que la rédaction de ces œuvres se concentre entre les années 1613 et 1615, c'est-à-dire au tout début de la production dramatique de l'auteur, ce qui pourrait attester l'hypothèse d'une production réduite, au profit des comedias. Une exception existe cependant : El laberinto de Creta. La pièce a manifestement été écrite en 1638 et son attribution à Tirso n'est pas encore totalement acquise bien que El Instituto de Estudios Tirsianos de l'université de Navarra l'intègre dans son édition des autos sacramentales de Tirso $^{2}$. Choisissant en quelque sorte de ne pas choisir dans un débat de spécialistes qui n'est pas l'objet de ce travail, j'ai bien intégré les relevés chiffrés à ceux effectués dans les autres pièces et exploité comme il se doit les résultats obtenus, tout en notant cependant que cette pièce ne met en jeu qu'un nombre très réduit d'allocutifs, ce qui limite la portée interprétative des relevés effectués à l'échelle de tous les autos.

Les six autos constituent donc bel et bien un corpus cohérent, et j'espère montrer ici que s'ils entretiennent une cohérence structurelle indiscutable avec l'ensemble du théâtre tirsien fondée sur une esthétique de l'alternance des pronoms et formules d'adresse, ils n'en restent pas moins des objets dramatiques originaux.

Comme le montre le tableau récapitulatif ci-dessous, les pièces concernées offrent une nette prédominance du tutoiement au détriment du voseo (à l'exception de El colmenero divino). On remarque également une limitation très nette dans la variété des formules d'adresse (limitées à cinq, en incluant le pronom vosostros, quand une bonne dizaine de tratamientos différents est couramment utilisée dans les comedias tirsiennes ou lopesques) ainsi que la présence relativement marquée du pronom de troisième personne él.

2. El laberinto de Creta, ed. I. Arellano, B. Oteiza y M. Zugasti, en Obras completas. Autos sacramentales, II, Madrid-Pamplona, Instituto de Estudios Tirsianos, 2000.

I. Arellano lui-même souligne l'incertitude quant à l'attribution de l'auto à Tirso de Molina. I. Arellano, Arquitecturas del ingenio. Estudios sobre teatro de Tirso de Molina, Madrid-Pamplona, Instituto de Estudios Tirsisanos, 2001. 
BULLETIN HISPANIQUE

\begin{tabular}{|l|c|c|c|c|c|}
\hline & $T u$ & Vos & El & $\begin{array}{c}\text { Alteza } \\
\text { Majestad }\end{array}$ & Vosotros \\
\hline El Colmenero divino (113) & $45,13 \%$ & $54,86 \%$ & & & \\
\hline La Madrina del Cielo (119) & $42,85 \%$ & $12,60 \%$ & $38,65 \%$ & & $5,88 \%$ \\
\hline No le arriendo la ganancia (152) & $46,60 \%$ & $34,10 \%$ & & $3,90 \%$ & $15,10 \%$ \\
\hline Los hermanos parecidos (110) & $51,81 \%$ & $24,45 \%$ & $8,18 \%$ & $1,81 \%$ & $12,72 \%$ \\
\hline La Ninfa del cielo (127) & $77,95 \%$ & $13,38 \%$ & & & $8,66 \%$ \\
\hline El Laberinto de Creta (54) & $74 \%$ & $22,3 \%$ & $3,7 \%$ & & \\
\hline
\end{tabular}

Ces simples constatations constituent en elles-mêmes une différence notable par rapport au phénomène d'alternance constaté dans les comedias, lequel est caractérisé par une polarisation très nette entre les pronoms $T u ́$ et Vos ainsi que par la présence de nombreuses autres formules d'adresse inscrites dans le plan sociolinguistique, comme le montre le tableau récapitulatif suivant :

\begin{tabular}{|c|c|c|c|c|c|c|c|c|c|}
\hline $\begin{array}{c}\text { Nombre total } \\
\text { des allocutifs }\end{array}$ & Tú & Vos & $\dot{E} l$ & Merced & Señoría & Exc. & Alteza & Maj. & Vosotros \\
\hline 28543 & 13228 & 11615 & 1129 & 196 & 121 & 213 & 461 & 156 & 1424 \\
\hline & $46,34 \%$ & $40,69 \%$ & $3,95 \%$ & $0,68 \%$ & $0,42 \%$ & $0,76 \%$ & $1,61 \%$ & $0,54 \%$ & $4,98 \%$ \\
\hline
\end{tabular}

Est-ce à dire que les autos s'affranchissent totalement d'un système interlocutif qui ne serait alors en vigueur que dans les comedias, les tratamientos se voyant investis d'un sens autonome, sans lien avec celui qui est le leur dans le théâtre profane ? Outre le fait qu'il est bien connu que les autos sont souvent considérés comme des "mini-comedias ${ }^{3}$ et

3. Les autos sacramentales, sont comme le rappelle Jean-Louis Flecniakoska: « una comedia de devoción reducida a un acto (...). [Es] un teatro de devoción, es decir un teatro que pretende ante todo conmover al mayor público posible. Es un teatro didáctico, con ambición lúdica, que, al igual que la poesía devota, utiliza, adoptándolo, un género pre--o co - existente. En la época de Lope es, antes que nada, una comedia devota (...) en un acto y de gran especularidad".

Jean-Louis Flecniakoska "¿Auto sacramental o poesía devota?", in Bruce Wardropper, 
qu'à ce titre une telle différenciation s'avèrerait plus que surprenante, il semble plutôt que les protocoles interlocutifs s'adaptent à un système dialogal sensiblement différent de celui mis en jeu dans les comedias, puisque sa finalité est ouvertement religieuse mais que, s'agissant d'un texte dramatique et fictionnel et non d'un simple catéchisme, ces protocoles ne sauraient s'affranchir d'une indéniable portée poétique. A cet égard, la comparaison avec les relevés concernant les dialogues entre humains et personnages surnaturels survenant dans 21 des 82 comedias de Tirso s'avère plus encourageant dans la recherche d'une cohérence interne à ce type de théâtre puisque nous y relevons près de $72 \%$ de tutoiement et un peu plus de $26 \%$ de vouvoiement.

Avant de poursuivre l'analyse proprement dite, un rapide aperçu du contenu des pièces est indispensable. Parmi les six autos qui nous intéressent ici, un n'est pas, à proprement parler, une pièce allégorique : La Madrina del cielo, dans la mesure où il offre une intrigue relativement proche de celle d'une comedia galante à thématique religieuse. Dans cette pièce, Dionisio, encouragé par son valet Doroteo, viole Marcela dont il est épris et se refuse à l'épouser une fois son forfait commis. La dame s'en remet alors au Christ à qui elle demande vengeance et qui la lui accorde " en personne " lors d'une apparition. Délaissant provisoirement la dame en détresse, la pièce nous met en présence de deux moines qui devisent en cheminant, Domingo (présenté par la didascalie comme Saint Dominique) et Chinarro. Ces deux religieux sont très rapidement attaqués par Doroteo et Dionisio qui leur intiment l'ordre de se dévêtir et de leur remettre tous leurs biens. Face à l'extrême humilité de Domingo, Dionisio est submergé par un scrupule jusqu'alors inconnu de lui et il oblige son très violent comparse à laisser partir les deux innocentes victimes. Domingo intercède alors auprès de la Vierge pour que celle-ci obtienne de son Fils qu'il ne cède pas au Diable, qui la réclame, l'âme de Dionisio, mais uniquement celle de Doroteo qui n'a jamais su témoigner d'aucune clémence. Le Christ accepte l'intercession de sa Mère et envoie l'Ange de la Miséricorde aider Dionisio dans son ultime combat contre le vice, le Diable lui-même. Sorti vainqueur de cette lutte, Dionisio se livre corps et âme au repentir. Survient alors Marcela affligée de ne pas obtenir la vengeance attendue. Après avoir rappelé à la Dame que tout repentir sincère entraîne nécessairement le pardon, le Christ procède au mariage de Dionisio et de Marcela.

Historia y crítica de la literatura española : Siglo de oro, Barroco, Barcelona, Editorial Crítica, 1983, p. 252. 
El laberinto de Creta, pour sa part, présente l'affrontement final entre Thésée et le Minotaure tout en assimilant le personnage du héros mythologique à la figure Christique et celui du roi Minos à celle du Démon ${ }^{4}$.

Les quatre autres pièces, sont, quant à elles, explicitement allégoriques, ne serait-ce que par le nom des protagonistes présentés par la didascalie initiale.

El Colmenero divino et La Ninfa del Cielo développent sensiblement la même intrigue : l'Âme, dupée par Le Monde dans la première et Le Péché dans la seconde, oublie son véritable Époux et est au bord de la chute avant d'être sauvée in extremis par la miséricorde du Christ.

Dans No le arriendo la ganancia, Honor, promis à Quietud mais séduit par Mudanza suit le roi Poder, et abandonne la vertu rurale pour la fièvre de la Cour où il se retrouve joué par les courtisans Interés, Recelo et Deseo.

Dans Los Hermanos parecidos enfin, l'Homme, choisi comme vice-roi du monde par Dieu lui-même, perd son trône après avoir cédé à son épouse Vanidad et après avoir goûté le fruit défendu. Rejeté de tous et condamné à périr, il est sauvé par son Frère, le Christ, qui se substitue à lui et se sacrifie pour le sauver.

Il est important ici de rappeler la vocation première de ces textes qui est celle de la représentation, évidence sans doute s'agissant de textes de théâtre, mais évidence à ne point perdre de vue dans le contexte de cette étude. De fait, là où le lecteur a pleinement conscience de la nature allégorique des entités mises en présence dans ces œuvres, le spectateur voit, avant tout, des personnages en chair et en os, et, qui plus est, vêtus de manière assez similaire à celle des personnages de comedias. La perception de ces pièces comme objets dramatiques plutôt que comme objets d'éducation religieuse ne doit donc pas être sous-estimée. Chaque intrigue, en effet, bien que religieuse, peut aisément acquérir une relative autonomie et devenir une simple " histoire ". La Ninfa del cielo d'ailleurs connaît un prolongement sous la forme de la comedia La Niña del cielo. Condesa bandolera y obligaciones del Cielo.

Chaque pièce offre donc une double perception (fût-elle simultanée), l'une littérale (l'histoire, l'action dramatique, l'aventure vécue par les personnages lesquels correspondent souvent, on le sait, aux archétypes de la Comedia) et

4. Ainsi que le rappelle I. Arellano, il est courant, au Siècle d'Or, de soumettre la mythologie à une interprétation allégorique comme on le voit, par exemple, dans Juan Pérez de Moya, Philosofía secreta, ed. de Carlos Clovería, Madrid, Cátedra, 1995.

Voir : Ignacio Arellano, Tirso de Molina, Textos e intertextos, Actas del congreso Internacional organizado por el GRISO y la Universidad de Parma, 7-8 de Mayo de 2001. Ed. Laura Dolfi, Eva Galar. Madrid, Revista Estudios, Pamplona, GRISO (Universidad de Navarra), Publicaciones del Instituto de Estudios Tirsianos ; 12, 2001, p. 51. 
l'autre tropologique (la mise en évidence des vices, des vertus et des passions qui constituent autant d'étapes que l'Âme doit parcourir dans son ascension vers Dieu). Ainsi que nous allons le monter maintenant, les pronoms et formules d'adresse constituent un des lieux privilégiés de la résolution de la tension entre ces deux perceptions d'un même texte.

Si nous considérons que dans chacune des pièces ici traitées deux pôles tentent d'agir sur un personnage central soit pour le sauver du péché en ce qui concerne les personnages bénéfiques soit pour l'y faire céder en ce qui concerne les personnages maléfiques, nous allons voir que ces deux familles de personnages ne bénéficient, de la part du dramaturge, ni du même traitement linguistique ni du même statut dramatique.

Le personnage central, le sujet, autour duquel et sur lequel agissent ou tentent d'agir ces opposants et ces adjuvants ${ }^{5}$ est soit l'Âme directement en tant qu'épouse du Christ, (qu'elle soit Abeja, Ariadna, ou Ninfa) soit une image parfois allégorique de l'homme, considéré comme pécheur, dans sa lutte pour la rédemption (Dionisio, Honor, ou Hombre).

La liste des personnages maléfiques, des opposants à la félicité du personnage central et de leurs complices, involontaires ou volontaires, n'est pas très longue. Il y a bien sûr le Démon, proprement dit, mais aussi toute une suite d'allégories des dangers qui guettent l'âme sur la voie de la félicité : la Vanité, le Désir, la Tromperie, l'Envie, le Monde, l'Ours, le Corps, le Péché, la Malice, le Mépris, le Pouvoir, l'Intérêt, la Versatilité. Ces différents personnages parlent entre eux, ils parlent également avec les personnages qu’ils ont en charge de pervertir et avec les personnages bénéfiques.

J'ai eu l'occasion au cours de ma thèse, de consacrer un développement spécifique aux dialogues mettant en jeu des personnages surnaturels entre eux ou en relation avec des humains. Le tableau des occurrences des divers types d'allocutifs concernant ces dialogues était le suivant :

\begin{tabular}{|c|c|c|c|c|c|c|c|}
\hline$\underline{\text { Total }}$ & Tú & Vos & Él/ella & Señoría & Excelencia & Alteza & Majestad \\
\hline 499 & 358 & 131 & 2 & 4 & 2 & 1 & 1 \\
\hline 21 pièces & $71,74 \%$ & $\mathbf{2 6 , 2 5 \%}$ & & & & & \\
\hline
\end{tabular}

5. Anne Uberfsfeld, Lire le théatre, Paris, Éditions Sociales, 1982, p. 62-63. 
La répartition, nous pouvons le constater, est semblable à celle observée dans les autos sacramentales, mais cette apparente similitude est néanmoins trompeuse pour ce qui concerne un protocole sur lequel je vais revenir plus loin.

D'une manière générale, dans les Comedias, le tutoiement est l'apanage des êtres surnaturels ${ }^{6}$ (divins ou diaboliques) qui n'usent quasiment jamais $\mathrm{du}$ vouvoiement que ce soit entre eux ou à destination des humains. Les humains pour leur part, oscillent entre un tutoiement de mépris, fortement marqué d'un point de vue sociodramatique, à destination des personnages diaboliques, et un vouvoiement respectueux ou un tutoiement d'adhésion et de soumission bienheureuse à destination des personnages divins.

Il en va de même dans les autos en ce qui concerne les dialogues entre humains ou personnages allégoriques liés à l'humanité et les êtres surnaturels bénéfiques. Ces dialogues sont finalement peu nombreux ${ }^{7}$ au regard du nombre total de dialogues dans ces pièces et se déroulent toujours selon le même protocole interlocutif ainsi que le montre le tableau récapitulatif cidessous :

\begin{tabular}{|c|r|c|}
\hline Titres & Tú & Vos \\
\hline El Colmenero divino & & \\
Abeja - Colmenero & 5 & 5 \\
Colmenero - Abeja & 12 & \\
\hline La Ninfa del cielo & & \\
Alma - Cristo & 2 & 5 \\
Cristo - Alma & 11 & \\
\hline La madrina del cielo & & \\
Marcela - Jesús & 4 & \\
Jesús - Marcela & & \\
\hline Doroteo - Virgen & 1 & \\
Virgen - Doroteo & & \\
\hline Los hermanos parecidos & 4 & \\
Hombre - Cristo & 8 & \\
Cristo - Hombre & & \\
\hline El Laberinto de Creta & 3 & \\
Ariadna - Teseo & 2 & \\
Teseo - Ariana & &
\end{tabular}

Le personnage humain (Dionisio dans La Madrina del cielo, Ninfa dans La Ninfa del cielo) ou le personnage allégorique réputé représenter l'Âme soumise à la tromperie du démon ou aux tentations du monde (L'Abeille de El Colmenero divino, l'Homme de Los hermanos parecidos) font alterner,

6. J'appelle dans ce contexte « êtres surnaturels » le Christ, la Vierge, un Ange, le Démon.

7. 82 allocutifs y sont mis en jeu. 
à l'adresse des personnages divins, tutoiement d'humilité et d'allégeance et vouvoiement de respect. Ce système interlocutif est conforme en tout point à celui mis en jeu dans les comedias. Il a ici pour fonction d'humaniser les personnages allégoriques placés en position d'objets du drame religieux qui se joue en leur attribuant le même protocole interlocutif qu'aux humains/ pécheurs, non seulement des autres autos, mais aussi des comedias édifiantes. Il est peu probable que l'actrice jouant "l'abeille ", allégorie de l'âme, dans $E l$ colmenero divino ait été effectivement vêtue en abeille lors de la représentation, mais ce que l'on peut affirmer avec certitude c'est que sa façon de s'adresser au divin Maître des abeilles est la même que celle de Juana s'adressant au Christ ou à son Ange Gardien ; en retour elle reçoit le même type d'adresses que la Sainte de la trilogie ${ }^{8}$. L'éloignement extrême par rapport au lecteurspectateur provoqué par la présence d'une figure allégorique (c'est aussi le cas pour le personnage nommé Honor de No le arriendo la ganancia) et limitant de fait la projection du spectateur sur un personnage et donc la portée cathartique de la pièce, se trouve ainsi compensé par la simplification du protocole interlocutif, ramené à un code commun auquel le lecteurspectateur peut aisément adhérer.

La figure divine quant à elle, s'en tient au tutoiement mis en ouvre dans les comedias. Ce tutoiement systématique, déconnecté de quelque code sociolinguistique ou sociodramatique que ce soit, de par son aptitude linguistique à réduire la distance entre locuteur et allocutaire d'une part et sa nature de tratamiento dramatique neutralisé d'autre part, inscrit ces figures divines dans un champ linguistique absolument transcendant, sans autre justification que la nature même du personnage à la caractérisation ${ }^{9}$ duquel il participe.

Pour résumer, les dialogues entre personnages divins et " pécheurs " (quelle que soit leur nature dramatique, humaine ou allégorique), sont entièrement conformes aux protocoles interlocutifs mis en jeu dans les comedias édifiantes et à thématique religieuse, protocoles qui participent linguistiquement d'une part de l'humilité du pécheur et de la supériorité transcendante du divin. Le schéma est donc bien celui d'une illusion dramatique orientée vers une

8. Il s'agit de la trilogie La Santa Juana, Trilogía hagio-gráfic, in Tirso de Molina, Obras dramáticas completas I, ed. Crítica por Blanca de los Ríos, Madrid, Aguilar, p. 723-909.

9. Nombreuses sont les paroles que Thérèse d'Avila, par exemple, rapporte avoir entendues de la bouche de Dieu, lequel s'adresse à elle en la tutoyant : "Hija yo quiero que mi sangre te aproveche y no hayas miedo que te falte mi misericordia; ¿Piensas hija, que está el merecer en gozar?; Aqui estoy, sino que quiero que veas lo poco que puedes sin mi. " in Obras de Santa Teresa de Jesús, editadas y anotadas por Silverio de Santa Teresa, T. I., Biblioteca Mística Carmelitana, Burgos, 1915, p. 57, 65, 81. 
forme de simplicité voire de limpidité étrangère à la complexité interlocutive et, au-delà, aux turpitudes du monde. Ce système interlocutif est presque éthéré, dégagé de la mise en forme dramatique des contentieux du Monde.

La remarquable économie de moyens mise en ouvre par Tirso dans les protocolesinterlocutifsquenousvenonsd'analysercontrastespectaculairement avec les protocoles mis en oeuvres dans les dialogues faisant intervenir des personnages maléfiques. Non seulement l'alternance des pronoms y est plus variée, mais en outre, ainsi que nous allons pouvoir le constater, l'alternance interne aux pronoms eux-mêmes y est plus importante.

Ainsi que le montrent les tableaux ci-dessous, la répartition des allocutifs est sensiblement différente de celle des dialogues précédemment évoqués, qu'il s'agisse des dialogues entre alliés du mal pour le premier tableau ou des dialogues entre personnages liés au mal et le personnage soumis à la tentation du mal pour le second:

\section{Tableau 1}

\begin{tabular}{|c|c|c|c|c|c|}
\hline Titres & $T u ́$ & Vos & $\dot{E} l$ & Alteza & Majestad \\
\hline $\begin{array}{l}\text { La Ninfa del Cielo } \\
\text { Pecado - Malicia } \\
\text { Malicia - Pecado }\end{array}$ & $\begin{array}{l}6 \\
6\end{array}$ & & 1 & & \\
\hline $\begin{array}{l}\text { Pecado - Deleite } \\
\text { Deleite - Pecado }\end{array}$ & $\begin{array}{l}2 \\
1\end{array}$ & & & & \\
\hline $\begin{array}{l}\text { No le arriendo la ganancia } \\
\text { Recelo - Poder } \\
\text { Poder - Recelo }\end{array}$ & 1 & 4 & & & \\
\hline $\begin{array}{l}\text { Poder - Mudanza } \\
\text { Mudanza - Poder }\end{array}$ & $\begin{array}{l}1 \\
1 \\
\end{array}$ & $\begin{array}{l}9 \\
4 \\
\end{array}$ & & 1 & 1 \\
\hline $\begin{array}{l}\text { La Madrina del cielo } \\
\text { Dionisio - Doroteo } \\
\text { Doroteo - Dionisio }\end{array}$ & $\begin{array}{l}4 \\
8 \\
\end{array}$ & & & & \\
\hline $\begin{array}{l}\text { El Colmenero divino } \\
\text { Oso - Mundo } \\
\text { Mundo - Oso } \\
\end{array}$ & $\begin{array}{l}1 \\
3 \\
\end{array}$ & & & & \\
\hline $\begin{array}{l}\text { Los hermanos Parecidos } \\
\text { Atrevimiento - Admiración } \\
\text { Admiración - Atrevimiento }\end{array}$ & 7 & 1 & 1 & & \\
\hline $\begin{array}{c}\text { Atrevimiento - Deseo } \\
\text { Engaño - Deseo } \\
\text { Codicia - Engaño } \\
\text { Engaño/Envi./Codicia/ Atrev. }\end{array}$ & 1 & $\begin{array}{c}2 \\
1 \\
1 \\
11\end{array}$ & & & \\
\hline TOTAL & $48(56,5 \%)$ & $33(39 \%)$ & 2 & 1 & 1 \\
\hline
\end{tabular}




\section{Tableau 2}

\begin{tabular}{|c|c|c|c|c|c|}
\hline Titres & $T \dot{u}$ & Vos & Él/Ella & Alteza & Majestad \\
\hline $\begin{array}{c}\text { La Ninfa del Cielo } \\
\text { Pecado - Alma } \\
\text { Alma - Pecado }\end{array}$ & $\begin{array}{l}4 \\
1\end{array}$ & $\begin{array}{l}3 \\
2\end{array}$ & & & \\
\hline $\begin{array}{l}\text { Voluntad - Alma } \\
\text { Alma - Voluntad }\end{array}$ & $\begin{array}{l}6 \\
3\end{array}$ & 1 & & & \\
\hline $\begin{array}{c}\text { No le arriendo la ganancia } \\
\text { Poder - Honor } \\
\text { Honor - Poder }\end{array}$ & 10 & $\begin{array}{l}6 \\
6 \\
\end{array}$ & & & \\
\hline $\begin{array}{c}\text { El colmenero divino } \\
\text { Mundo - Cuerpo } \\
\text { Cuerpo - Mundo }\end{array}$ & 3 & 6 & & & \\
\hline $\begin{array}{l}\text { Mundo - Abeja } \\
\text { Abeja - Mundo }\end{array}$ & $\begin{array}{l}5 \\
2\end{array}$ & & & & \\
\hline $\begin{array}{l}\text { Oso - Abeja } \\
\text { Abeja-Oso }\end{array}$ & 1 & & & & \\
\hline $\begin{array}{l}\text { Cuerpo-Abeja } \\
\text { Abeja-Cuerpo }\end{array}$ & $\begin{array}{l}3 \\
8\end{array}$ & 10 & & & \\
\hline $\begin{array}{l}\text { Los Hermanos parecidos } \\
\text { Atre./Engaño/Temor - Hombre } \\
\text { Hombre-Atrev./Engaño/Temor }\end{array}$ & $\begin{array}{c}15 \\
2 \\
\end{array}$ & 1 & 1 & & \\
\hline \begin{tabular}{|l|} 
Total \\
\end{tabular} & $63(63,5 \%)$ & $35(35,5 \%)$ & 1 & & \\
\hline
\end{tabular}

Ainsi que nous pouvons le constater, l'augmentation du nombre de vos d'une part et l'apparition de la troisième personne et de formules d'adresse beaucoup plus marquées d'un point de vue sociolinguistique d'autre part caractérisent ces dialogues. De fait, le contexte d'énonciation entraîne une variation interne du sens des allocutifs convoqués - rendue possible par le jeu sur la double compétence sociolinguistique et poétique de ces allocutifs tant il est vrai, comme le rappelle Anne Ubersfeld que la didascalie détermine une pragmatique : les conditions concrètes d'usage de la parole ${ }^{10}$. Le tutoiement reprend, dans ces dialogues, sa valeur de proximité sociodramatique ou affective (entre une dame et son galant par exemple) de vecteur privilégié des relation maître/valet et valet/maître (comme entre Oso et Mundo) ou d'expression d'une supériorité dans la hiérarchie des dramatis personae comme entre Mundo et le rustique Cuerpo (ou encore le tutoiement royal que convoque Poder envers Honor). Le voseo pour sa part, voit réactivée sa valeur galante et palaciega caractéristique des dialogues galants/dames de la comedia tirsienne (entre Poder et Mudanza), sa valeur de politesse rustique

10. Lire le théâtre, p. 21. 
et peu adroite (comme dans l'adresse du bobo Cuerpo aussi bien à Mundo qu'à $A b e j a$ ) ou de politesse moyenne, voire méprisante, entre égaux, comme c'est le cas entre les comploteurs Atrevimiento, Deseo, Engaño et Codicia ou entre la traîtresse Voluntad et le fidèle Entendimiento de La Ninfa del Cielo.

Les dialogues entre Pecado et Alma/Ninfa dans La Ninfa del cielo offrent une illustration probante de ces constats. Pecado, a quitté la ville, estimant que les proies y sont trop faciles à capturer, pour la campagne. Là, il rencontre Alma/Ninfa qu'il s'emploie à séduire, non sans un certain succès dans un premier temps. La rencontre et la scène de séduction, plus ramassées que dans les comedias, mettent cependant en jeu les mêmes protocoles :

$\begin{array}{ll}\text { Pecado : } & \begin{array}{l}\text { Hermosisima señora, } \\ \end{array} \\ & \text { Un cazador soy perdido } \\ & \text { Desde que salió la aurora, } \\ & \text { Pero me he arrepentido } \\ & \text { Porque ya mi pecho adora } \\ & \text { Vuestro rostro angelical. } \\ & \\ \text { Al... } & \text { Vuestro talle obliga } \\ & \text { A serviros y hospedaros. } \\ & \text { ¿Quién sois para que yo diga } \\ & \text { que me ocupé en regalaros? }\end{array}$

puis, en dépit des remontrances qu'elle subit de la part de ses serviteurs Memoria et Entendimiento, mais encouragée par sa servante Voluntad, Alma se livre à Pecado en ces termes:

$\begin{array}{ll}\text { Alma: } & \text { Pues jalto! mi mano es esta } \\ & \text { Y a tu gusto desde hoy, } \\ & \text { Esposo, estaré dispuesta. } \\ \text { Pecado : } & \text { Yo soy tuyo. }\end{array}$

L'alternance mise en jeu dans cette scène hautement symbolique est bel et bien celle des scènes galantes. Mais à notre sens, il ne s'agit pas uniquement ici de la passive reconduction d'un protocole mécaniquement appliqué, issu des archétypes interlocutifs de la comedia. De fait, l'allégorie, la leçon dogmatique se voit ancrée, notamment par les allocutifs, structure profonde du dialogue, dans une représentation dramatique qui est identique à celle du théâtre profane de divertissement. Ce traitement galant de la chute de l'Âme, son inscription dans un code dramatique éminemment terrestre et 
mondain, inscrit de fait le péché, la faute, le mal dans le domaine humain. Certes, le thème est grave, l'allégorie est présente, mais en étant traitée selon un protocole interlocutif propre à ce qui constitue une scène de genre du teatro nuevo elle ne décolle jamais de la bassesse terrestre, elle ne saurait atteindre la "neutralité éthérée " que je soulignais plus haut à propos des dialogues mettant en jeu un personnage divin ${ }^{11}$. Au reste, il est significatif que lorsque l'Âme, enfin consciente de sa faute, revient vers le Christ, le protocole mis en jeu parte d'un tutoiement de transcendance pour ensuite adopter un vouvoiement d'humilité absolue, qui renoue avec son utilisation médiévale de tratamiento royal. Ce renversement linguistique est aussi un renversement géographique : après s'être abaissé jusqu’à la trivialité la plus bassement terrestre, le texte (et l'Âme) s'élève à nouveau vers une transcendance céleste.

En réalité, les dialogues entre pécheur/pécheresse et les différents avatars du péché (Poder, Pecado, Mundo) ne sont pas les seuls à voir leur protocole interlocutif s'inscrire nettement dans un espace référentiel qui est celui du teatro nuevo. De fait, tous les autres types de dialogues le font, qu'il s'agisse des dialogues entre serviteurs du mal et serviteurs du bien (T1), des dialogues entre serviteurs du bien et pécheur/pécheresse (T2), ou des dialogues entre serviteurs du mal et pécheur/pécheresse (T3) ainsi que le montrent les tableaux récapitulatifs suivants :

\section{Tableau 1}

\begin{tabular}{|c|c|c|}
\hline & Tú & Vos \\
\cline { 2 - 3 } La Ninfa del Cielo & & \\
Entendimiento - Voluntad & 2 & 1 \\
Voluntad - Entendimiento & & 1 \\
\hline Voluntad - Memoria & & \\
Memoria - Voluntad & 3 & \\
\hline El colmenero divino & & 5 \\
Placer - Oso & 2 & \\
Oso - Placer & 2 & \\
\hline
\end{tabular}

11. C'est un effet identique que produit l'alternance des pronoms entre Honor l'ambitieux courtisan et Poder, le puissant souverain. En effet, alors que Poder use subtilement d'un voseo tantôt flatteur, tantôt méprisant et d'un tutoiement de connivence caractéristique des dialogues entre un roi et son favori, Honor lui, décline très classiquement la gamme allant de Majestad et Alteza, au voseo du courtisan et au tutoiement du valido. 
Tableau 2

\begin{tabular}{|l|c|c|c|c|}
\hline \multicolumn{1}{|c|}{$\begin{array}{l}\text { La Ninfa del cielo } \\
\text { Entendimiento - Alma }\end{array}$} & Tú & Vos & Él/Ella & Vuestra señoría \\
\cline { 2 - 5 } Alma - Entendimiento & 16 & & & \\
\hline $\begin{array}{l}\text { Memoria - Alma } \\
\text { Alma - Memoria }\end{array}$ & 14 & & & \\
\hline $\begin{array}{l}\text { No le arriendo la ganancia } \\
\text { Escarmiento - Honor }\end{array}$ & 1 & & & \\
Honor - Escarmiento & 1 & 7 & & \\
\hline $\begin{array}{l}\text { Acuerdo - Honor } \\
\text { Honor - Acuerdo }\end{array}$ & 10 & 4 & 1 & \\
\hline $\begin{array}{l}\text { Quietud - Honor } \\
\text { Honor - Quietud }\end{array}$ & 3 & 1 & & \\
\hline $\begin{array}{l}\text { El colmenero divino } \\
\text { Placer - Abeja } \\
\text { Abeja - Placer }\end{array}$ & 4 & 1 & & \\
\hline
\end{tabular}

\section{Tableau 3}

\begin{tabular}{|l|c|c|c|}
\hline & $T u$ & Vos & Él/Ella \\
\cline { 2 - 4 } $\begin{array}{l}\text { La Ninfa del cielo } \\
\text { Voluntad - Alma } \\
\text { Alma - Voluntad }\end{array}$ & 6 & 1 & \\
\hline $\begin{array}{c}\text { No le arriendo la ganancia } \\
\text { Poder - Honor }\end{array}$ & 3 & & \\
Honor - Poder & 10 & 6 & \\
\hline El colmenero divino & 3 & 6 & \\
Cuerpo - Abeja & 8 & 10 & \\
Abeja - Cuerpo & & & \\
\hline $\begin{array}{c}\text { Los Hermanos parecidos } \\
\text { Atre./Engaño/Temor-Hombre }\end{array}$ & 15 & 1 & 1 \\
Hombre-Atrev./Engaño/Temor & 2 & & \\
\hline
\end{tabular}

Ainsi, lorsque dans No le arriendo la ganancia, Honor, aveuglé par son ambition et son éphémère réussite à la Cour ne reconnaît pas son frère Acuerdo ni sa cousine Quietud, l'alternance entre voseo (de politesse distante ou de mépris) et tutoiement (de proximité familiale ou de dédain) chez chacun des personnages suit fidèlement la courbe de l'intensité de l'affection familiale que chacun éprouve pour l'autre. L'ironie le dispute à la tristesse lorsque Quietud, excédée par la suffisance d'Honor, s'exclame :

Para vuestra señoría

Es toda la dicha y gala. 
Et plus loin:

\author{
Señor, pelota de viento, \\ Vos haréis algunas faltas \\ $Y$ os romperá la fortuna \\ $[\ldots]$
}

La formule d'adresse vuestra señoría justifiée du point de vue de Honor, est excessive du point de vue de Quietud; le pronom vos, apte à exprimer une politesse palaciega de bon aloi, héritée de l'usage sociolinguistique, est aussi une forme de mépris lié à l'éloignement entre locuteur et allocutaire. Les deux allocutifs sont, bien sûr, la clé de voûte de l'ironie cinglante du personnage de Quietud. En dernière analyse, ils rattachent également toute la scène ${ }^{12}$ à une scène de genre comme il s'en produit de nombreuses dans les comedias tirsiennes.

Cela étant, l'inscription de l'allégorie au cœur même d'un protocole interlocutif et donc, plus globalement, d'un schéma théâtral caractéristique d'une comedia de palais trouve son aboutissement, voire son dépassement dans l'une des dernières adresses de la pièce. Tel le fils prodigue, trahi par le roi Poder et par son épouse Mudanza, Honor décide de faire amende honorable et de rentrer dans son village. Il y est accueilli par son frère, Acuerdo qui s'apprête à épouser Quietud:

\title{
¡Detente hermano infelice! \\ [...] \\ Tu hermano soy, el Acuerdo \\ [...] \\ Tu dolor y enmienda basta;
}

Le maintien du tutoiement s'inscrit dans le protocole habituel des dialogues entre frères et sœurs. Or, un changement spectaculaire se produit après qu'Honor a demandé la raison du banquet qu'il voit se préparer et après que Quietud lui a appris qu'il s'agissait de ses noces avec Acuerdo. Ce dernier en effet lance sa dernière adresse de la pièce :

12. La scène est précédée d'un dialogue entre Quietud, Acuerdo et Recelo, un paysan devenu serviteur d'Honor à la Cour et qui remplit ici le rôle du gracioso. Comme dans la comedia, c'est le tutoiement systématique qui caractérise ces échanges, de même que les échanges entre Recelo, le gracioso et Honor, son maître. On peut souligner au passage que le rôle dévolu par l'Auto au personnage nommé Recelo peut constituer une forme de métadiscours "à distance " sur la principale "qualité " attribuée au gracioso dans la comedia cette fois. A ce titre, les autos fournissent une intéressante grille de lecture et de compréhension des différents actants des comedias. 


\author{
Honor, lavaos en la fuente \\ De la penitencia clara, \\ Que quita manchas de culpas \\ $Y$ da aguamanos de gracias \\ Porque comáis con nosotros.
}

Le passage au voseo dans une scène clairement eucharistique alors que jamais les échanges n’ont dérogé aux protocoles interlocutifs mis en jeu par la comedia tirsienne, distingue soudainement l'adresse, la surligne en quelque sorte, et la détache de la référence dramatique exploitée jusqu’alors pour l'élever vers celle de l'allégorie. La transcendance, jusque là rendue sinon impossible du moins difficile à percevoir dans une oeuvre usant de tous les codes d'un autre type de fiction dramatique, trouve enfin sa forme d'expression la plus spectaculaire puisqu'elle lie de manière indissociable références lexicales (la fuente clara, la penitencia, las manchas, la culpa, las gracias) et choix des pronoms d'adresse. L'illusion dramatique change de registre et le lecteur-spectateur peut prendre part à la scène d'action de grâce.

\title{
Conclusion
}

Ainsi que nous pouvons le constater, l'étude, même partielle, des protocoles interlocutifs des autos sacramentales tirsiens révèle - sans surprise une proximité structurelle entre autos et comedias considérés comme genres dramatiques, mais également des décalages subtils permettant précisément d'ancrer chacune des deux catégories dramatiques dans leur propre aire référentielle. Létude des autos sous l'angle des protocoles interlocutifs qui y sont convoqués permet d'affirmer que l'alternance des pronoms d'adresse y joue un rôle structurant similaire à ce que nous avons observé dans les comedias : elle participe de fait de la définition des personnages ou plus exactement des statuts dramatiques, étant entendu que chaque type de dialogue jouit de son propre protocole (alternance réduite au tutoiement et au vouvoiement entre les sujets et les personnages surnaturels bénéfiques; alternance intégrant un plus grand nombre de formules d'adresse entre les sujets et les personnages maléfiques ou leurs divers adjuvants). Au-delà de cette fonction, elle inscrit également les entités non anthropomorphes (adjuvants du Bien, personnages surnaturels maléfiques et adjuvants du Mal) dans un cadre référentiel qui est celui des archétypes de la comedia soulignant par ricochet, la valeur morale des personnages de comedia, bien 
que cet aspect des choses ne rentre pas dans les limites fixées à ce travail. Les pronoms d'adresse sont donc bel et bien le lieu où se résout la tension entre création poétique et discours théologique.

Cette double efficacité de l'alternance des pronoms et formules d'adresse constitue à la fois l'originalité du système mais également, au final ce qui le différencie de ce qui est mis en jeu dans les comedias. A ce titre, elle a donc également le mérite, plus métatextuel sans doute, de signaler un de ces décalages qui font qu'un auto ne puisse sans doute pas totalement être considéré, à proprement parler, comme une comedia en un acte. 